\title{
Gas Chromatography And Mass Spectrometry in Lipid Profiling of Allergy causing Food Materials
}

\author{
Elakpo Bridget Pabor $^{1^{*}}$ and Ekpe EL ${ }^{2}$ \\ ${ }^{1}$ School of Medicine, University of Swansea, Wales, United Kingdom \\ ${ }^{2}$ Department of Chemical Pathology/Immunology, University of Calabar, Calabar, Nigeria \\ Received 04 Feb 2018, Accepted 08 April 2018, Available online 11 April 2018, Vol.6 (March/April 2018 issue)
}

\begin{abstract}
Background: Food allergy is a major problem in westernized societies and the prevalence has been shown to have increased over the years. Peptide molecules have been known to be implicated in food allergy, however, peptides are not the only molecules recognized by $T$ cells in relation to food allergy. Lipids have been shown to stimulate an allergic response via certain pathways and this study aims to assess food allergy from phospholipids obtained from cypress grains.

Method: Investigation was focused on profiling lipids and comparing them based on their fatty acid and phospholipid composition by combinatorial analysis of peptides and proteins using Matrix Assisted Laser Desorption lonization (MALDI) and Gas chromatography/mass spectrometry analysis and also to create a phylogenetic relationship between samples and see if their overlap account for any known report of allergy.

Results: Here, we report that regardless of the classification system of samples (i.e. seed, nuts, and legumes), in terms of fatty acid and phospholipid compositions, some samples with similar origin differ from one another and unrelated families were closely related in the cluster that cause food allergy. Phospholipids were observed to be predominant in the nut species compared to fatty acids and this may have roles in stimulating an allergic response.

Conclusion: Food allergy may certainly be linked to lipid molecules.
\end{abstract}

Keywords: Allergy, lipid, peptides, mass spectrometry, fatty acid, chromatography

\section{Introduction}

Food allergy is a condition whereby individuals develop an aberrant immunologic reaction characterized by immunoglobulin $E(\operatorname{IgE})$ antibodies to harmless food antigens ${ }^{1}$. Generally, these reactions are immediate and occur each time food is ingested even in small amounts ${ }^{2}$. This may be due to factors inherent in foods such as toxic contaminations, pharmacologic properties of food or even the characteristics of the sensitized individuals such as metabolic disorders or idiosyncratic responses making the individual produce IgE antibodies against the innocuous antigens ${ }^{3,4}$.

In westernized societies, food allergy is a major public health problem and occasionally results in fatalities with about $90 \%$ of anaphylactic deaths resulting from peanut/tree nut reactions in the United States. Over $80 \%$ of children are allergic to foods such as cow's milk, egg, wheat and other cereal grains, though they develop tolerance to their allergy over time ${ }^{5}$. Allergies to peanut (a legume) and tree nut (example; walnut, pecans and

*Corresponding author's ORCID ID: 0000-0002-4205-7916 DOI: https://doi.org/10.14741/ijmcr/v.6.2.10 almond) is characterized by a high frequency of lifethreatening anaphylactic reactions and patients rarely become tolerant to them and face a lifelong persistence $^{3,6}$.

Although peanuts and tree nuts are associated with some health benefits such as reduced incidence of coronary heart disease and cancer they are also one of the leading causes of fatal and near-fatal food-induced allergic reactions ${ }^{7,8,9}$

\section{Mechanisms of allergic response}

One of the mechanisms that stimulate an allergic response is the IgE-mediated immune mechanism. Here, ingested food (allergic proteins) are acted upon by specific IgE antibodies residing on mast cells and basophils and the activation of these cells result in the release of a number of potent mediators and cytokines ${ }^{3}$. Mast cells are the key effector cells of allergic inflammation and they are located at strategic sites such as the skin, and the vascular and mucosal barriers ${ }^{10}$. When food allergens are taken up by dendritic cells (DCS) or antigen presenting cells (APCs), peptides are 
presented to T cells (TH2 variety) leading to the activation of $B$ cells that eventually mature into IgE-producing plasma cells and on subsequent exposure, the crosslinking of surface-bound IgE by allergen triggers mast cell activation and the release of histamines and other chemical mediators to stimulate the early phase of type I hypersensitivity; allergic reactions due to the production of IgE against innocuous antigens ${ }^{1,4,10}$. For this process to occur, individuals must first be exposed to the allergen and then become sensitized to it by producing $\lg \mathrm{E}$ antibodies and re-exposure to the allergen causes allergic reactions ${ }^{1,4}$. Mast cell activation requires access of the allergen into the tissue and input from the adaptive immune system. The process does not only require the synthesis of specific IgE by B cells (regulated by interleukin-4 and 13) but also mast cell priming by IL-4 for enhanced mediator release is required ${ }^{11}$. Agea et al using phospholipids extracted from cypress grains showed that lipid antigens by means of cluster of differentiation(CD) 1 proteins were able to stimulate an allergic response ${ }^{12}$. CD1 antigen presentation pathway involves both the innate and adaptive immune system.

\section{GC-MS and MALDI analysis of lipids}

Gas chromatography/mass spectrometry (GC/MS) is a powerful technique for the analysis of metabolites and it is useful in the rapid determination of fatty acid structures. In metabolomics, the metabolite profile can be compared and contrasted using (GCMS) analysis ${ }^{13,14}$. However, lipid extracts from biological sources are very complex hence it is important to perform a separation process prior to analysis.

Analysis of peptides and proteins using Matrix Assisted Laser Desorption Ionization (MALDI) Mass Spectrometry has been successful over the years however, in lipidomics it is still lacking. Woods and Jackson successfully studied lipid composition and distribution in a rat's brain and MALDI was used in the experiment to directly probe tissues in order to study their structural components. It was concluded that MALDI was well suited for biomolecular analysis in tissue because of its high sensitivity, large tolerance of salts and other contaminants and also a wide mass range with little fragmentation ${ }^{15}$. In order to understand the molecular properties that may classify lipids as allergens particularly in anaphylactic reactions, there is need to create a phylogenetic relationship between lipid contents derived from different nut species and study whether this relationship represents a known association between allergy prevalence of the studied foods and this will be achieved by profiling these lipid components by combinatorial GCMS and MALDI analysis. This study aims at analysing fatty acid composition and saturation by GCMS analysis and studying phospholipid composition by MALDI mass spectrometry analysis and correlating these lipids contained in nut/legumes to allergic responses.

\section{Materials and methods}

Lipids were extracted from these samples using Folch extraction method whereby $0.5 \mathrm{~g}$ of each sample was mixed using a motor-pistol (purchased from a local shop) with $5 \mathrm{ml}$ of $2: 1$ chloroform/methanol $\left(\mathrm{CHCl}_{3} / \mathrm{MeoH}\right)$ solution and centrifuged at $4000 \mathrm{rpm}$ for $10 \mathrm{mins}$.

\section{MALDI Analysis}

After centrifugation, the samples were filtered under pressure using a vacuum pump and $0.5 \mathrm{ml}$ aliquots were transferred into Eppendorf and spun to dryness. $1 \mu \mathrm{l}$ of each sample was placed in a MALDI plate with $1 \mu$ of 2,5 dihydrobenzoic acid (DHB) $(10 \mathrm{mg} / \mathrm{ml}$ in methanol with $0.1 \mu \mathrm{l}$ TFA) and $1 \mu \mathrm{l}$ of P-Nitroalanine $(10 \mathrm{mg} / \mathrm{ml}$ in $2: 1$ chloroform/methanol) on separate sides of the plate and this was taken for MALDI analysis. The sample was analysed by a Voyager DE-STR MALDI MS (Applied Biosystems) used in reflector mode with an acceleration voltage of $20000 \mathrm{~V}$, a grid voltage of $95 \%$ of the acceleration voltage and a delay time of 150 nanoseconds (ns). Laser intensity was manually altered in order to obtain a sufficient signal. In the investigation both $\mathrm{DHB}$ and P-Nitroalanine were used as the matrix for the detection of positive and negative ions respectively.

\section{GC/MS Analysis}

After centrifugation, the top layer (waste) was removed and the bottom layer was transferred into a screw layer which was blown down to minimum volume under pressure. Following the addition of $3 \mathrm{ml}$ of $3 \mathrm{M}$ hydrochloric acid/methanol $(\mathrm{Hcl} / \mathrm{MeoH})$ the sample was vortexed and kept in the oven at $90{ }^{\circ} \mathrm{C}$ for an hour. At the completion of this step, the sample was transferred into a clean boiling tube with the addition of $6 \mathrm{ml}$ of water and $3 \mathrm{ml}$ hexane and it was vortexed and allowed to settle. Again the top layer (hexane) was removed and transferred into a clean tube. This step was repeated twice and the hexane fractions were combined and blown to minimum volume under a stream of nitrogen which was then made up to a millilitre for GC analysis.

GCMS analysis involved the injection of $1 \mathrm{ul}$ sampler onto Agilent GCMS system. The column was HP-5MS (30metres* 250uM and a $0.25 \mathrm{uM}$ internal diameter). The carrier gas was Helium at a flow rate of $1 \mathrm{ml} / \mathrm{min}$. The temperature of the $\mathrm{GC}$ oven was set initially at $60{ }^{\circ} \mathrm{C}$ for 1 minute then increased by 10 degrees per $\min$ to 180 degrees and then by 4 degrees per min to 300 degrees.

The eluent was passed to a 5975 MSD mass selective detector which had an ion source temperature of 250 degrees centigrade and used an electron ionisation source to generate ions. All chemicals for GCMS and MALDI were purchased commercially, unless stated otherwise.

\section{Statistical analysis}

Both MALDI and GCMS data were incorporated into excel spreadsheet separately. For GCMS, the retention times 
and peaks were used to obtain the specific area of each sample by dividing each peak by the total number of peak area multiplied by 100 . This was done in replicates of four for each sample. The results were used to calculate the mean and standard deviation. Statistical comparison of samples was calculated by the Mann-Whitney $U$ test and $p$ test of $\leq 0.05$ was considered significant for statistical evaluation of the differences and similarities of the samples. Dendrograms were obtained by Hierarchical cluster analysis using the mean of each sample done in replicates (four for each). Any error bars in the results section figures represents standard deviations of each respective data. All statistical computations were done using SPSS and excel.

\section{Results}

Using the mean of samples done in replicates, a dendrogram was produced whereby samples were classified in the 10 groups highlighted in red based on the similarities of their fatty acid composition (see figure 1 ). The mean samples were obtained by using GCMS data analysis and SPSS was used to produce a dendrogram. The dendrogram is a representative diagram showing the relationship between nut species that were similar and /or dissimilar in fatty acid compositions.

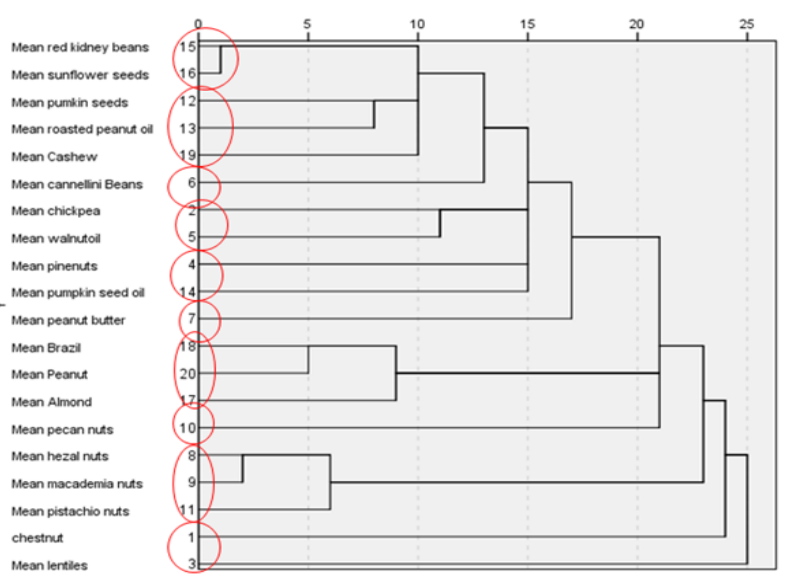

Figure 1 Dendrogram-Using of Single Linkage. The nearer they are, the more similar and the further they are they more different. Groups highlighted in red shows the level of similarity between samples

\section{Statistical analysis of the most common fatty acid compositions}

The mean of the various variables was used to generate bar charts where their standard deviations were represented as error bars. The graph shows that these fatty acids were present in most species, however they were absent in some. The number of individual repeats $(n)$ is four and the error bars show the range between the highest and lowest mean value. The mean was represented in percentages because it was calculated by using the specific areas (represented as \%) of each sample.

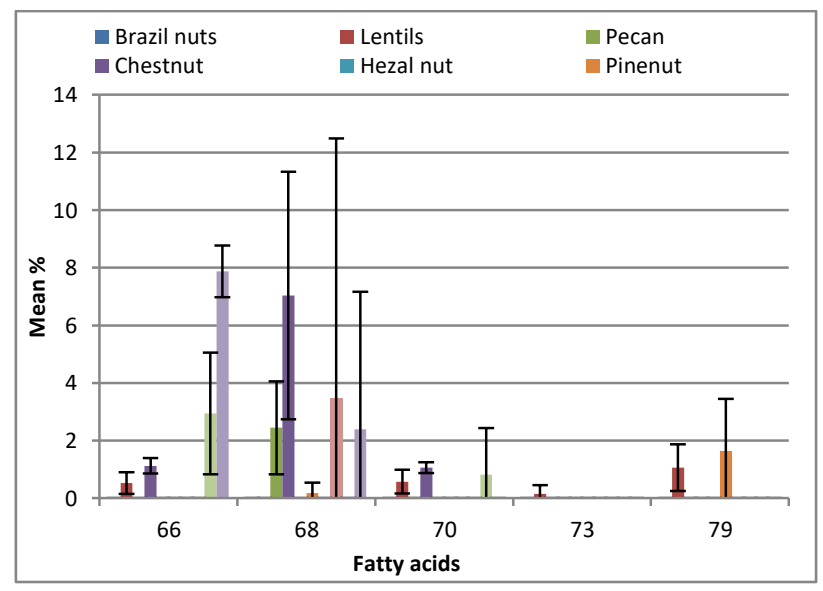

Figure 2 Analysis of the most common variables from GCMS

\section{Discussion}

Phospholipids were identified using their $\mathrm{m} / \mathrm{z}$ (mass-tocharge ratio) values and $\mathrm{m} / \mathrm{z}$ within the range of 758 to 790 was of a particular interest as they clearly showed the differences and similarities between phospholipid species. Our approach in using combinatorial GCMS and MALDI in the identification of lipids species present in different nut species was successful as it provided significant relationships between species by allowing easy comparison. The idea was to show diversity in terms of representing data and to confirm validity of the results. The comparison of lipid components using GCMS provided information on the number of double bonds and their locations but it gave no information about their origin whereas MALDI analysis provided information on the origin of lipids and number of double bonds, however it gave no information on the locations of the bonds. Another interesting finding from our results showed that results from MALDI and GCMS were different. The statistical analysis proved that there were more phospholipids in nut species compared to fatty acids and several conclusions can be drawn from this. First, we can say that the more phospholipids in a particular sample the higher the chances of its allergenicity and the same conclusion was made when we compared our data with Agea et al. They proved that phospholipids stimulated an allergenic response in cypress pollen especially PC 16:0 and PC 18:2 as they were the most predominant component of the cypress pollen. However, there might be drawbacks as our result might not be a strong positive due to some experimental errors that may have occurred. Our study has provided information concerning the identification of lipid species specifically by detecting their retention times (GCMS) and masses (MALDI) and the generation of a phylogenetic relationship based on this data. This information is promising in terms of understanding antigenic lipid presentation and it may 
suggest that samples with similar fatty acid composition will be recognized and presented by similar antigenic lipids whereas samples with different fatty acid composition will be recognised and presented by different antigenic lipids. The importance of this suggestion is to enable scientists understand how the CD1 pathway works, since our knowledge concerning it is still lacking.

\section{Future work}

Although we were able to create a phylogenetic relationship of lipid species, we were not able to show that these lipids can stimulate an allergic response. For this to be possible, we would need have sensitized individuals, whereby a skin prick test could be conducted and a positive response would show allergenicity whereas a negative response will be discarded. These are potential key points for future studies.

\section{References}

[1]. Otsu, K., S.C. Dreskin. Peanut Allergy: An Evolving Clinical Challenge. Discovery Medicine 65: 319-328;2011.

[2]. Lee, L.A., Burks AW, (2006) Food allergies: Prevalence, molecular characterization, and treatment/prevention strategies. Annual Review of Nutrition 26: 539-565;2006.

[3]. Sampson, H.A., L. Mendelson \& J.P. Rosen, (1992) Fatal and near-fatal anaphylactic reactions to food in children and adolescents. New England Journal of Medicine 327: 380384.
[4]. Murphy, M. K.Janeway's Imunobiology $8^{\text {th }}$ Ed. In:Garland Science, Taylor \& Francis group LLC: New york pp 371373;2006.

[5]. Nowak-Wegrzyn, A. \& H.A. Sampson. Future therapies for food allergies. J. Allery Clin. Immunol 127: 558-575;2011.

[6]. Bock, S.A. \& F.M. Atkins.The natural-history of peanut allergy. J Allergy Clin. Immunol 83: 900-904;1989.

[7]. Emilio R. Health benefits of nut consumption. Nutrients 2(7):652-682;2010

[8]. Griel, A.E. \& P.M. Kris-Etherton.Tree nuts and the lipid profile: a review of clinical studies. British Journal of Nutrition 96: S68-S78;2006

[9]. Sicherer, S.H., Munoz-Furlong, A., Burks , A.W \& H.A. Sampson. Prevalence of peanut and tree nut allergy in the US determined by a random digit dial telephone survey. $J$. Allery Clin. Immunol103: 559-562;1999.

[10]. Bradding, P., A.F. Walls \& S.T. Holgate, (2006) The role of the mast cell in the pathophysiology of asthma. J. Allery Clin. Immunol117: 1277-1284;2006.

[11]. Bischoff, S.C. Role of mast cells in allergic and non-allergic immune responses: comparison of human and murine data. Nature Reviews Immunology 7: 93-104;2007.

[12]. Agae, E., Russano, A., Bistoni, O., Mannucci, R., Nicoletti I., Corazzi, L et al. Human CD1-restricted T cell recognition of lipids from pollens. J Exp Med 295-308;2005

[13]. Roberts, D.L, Mccombie, G., Titman, C.M \& Griffin, L.J. A matter of fat: An introduction to lipid profiling methods. J. Chrom. 871: 174-181;2008

[14].Woods, A.S, Jackson N.S. Brain tissue lipidomics: Direct probing using matrix assisted laser desorption/ ionization mass spectrometry. J. AAPS. 8: E391- E395;2006. 